\title{
CARACTERIZAÇÃO DA OBESIDADE EM PACIENTES COM INFARTO DO MIOCÁRDIO
}

\author{
Roberta Cunha Rodrigues Colombo \\ Olga Maimoni Aguillar \\ Maria Cecília Bueno Jayme Gallani ${ }^{3}$ \\ Cláudio Alexandre Gobatto ${ }^{4}$
}

Colombo RCR, Aguillar OM, Gallani MCBJ, Gobatto CA. Caracterização da obesidade em pacientes com infarto do miocárdio. Rev Latino-am Enfermagem 2003 julho-agosto; 11(4):461-7.

Este trabalho objetivou caracterizar a obesidade em pacientes infartados. Os dados foram obtidos junto a 43 pacientes de um hospital universitário. Constatou-se que 69,7\% dos sujeitos possuíam Índice de Massa Corporal (IMC) maior que o

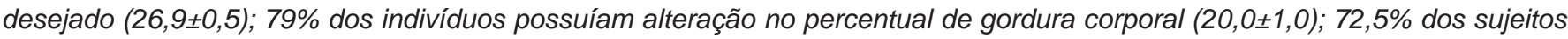
apresentavam razão cintura quadril acima do desejado (0,97士0,03); a circunferência da cintura (CC) esteve alterada em 64\% dos sujeitos $(97,3 \pm 1,8)$. Como a CC correlacionou-se significativamente com o IMC e percentagem de gordura corporal, nesse grupo estudado, sugere-se a determinação do IMC e da CC para avaliação da obesidade em nível ambulatorial.

DESCRITORES: obesidade; infarto do miocárdio; reabilitação

\section{OBESITY IN PATIENTS WITH MYOCARDIAL INFARCTION}

This study aimed to characterize obesity in infarcted myocardial patients. Data were collected from 43 patients at a University Hospital. It was observed that the Body Mass Index (BMI) was above the desired level for 69.7\% (26.9 0.5 ) of the subjects; the body fat percentage had altered in $79 \%$ (20.0 1.0$)$ of cases; the waist/hip (W/H) ratio was above normal in $72.5 \%$ $(0.97 \pm 0.03)$ of cases and the waist circumference (WC) had altered in 64\% (97.3 \pm 1.8$)$ of the patients. Since the correlation for $B M I, W C$ and fat percentage was significant, the use of BMI and WC was suggested for evaluating obesity in outpatients.

DESCRIPTORS: obesity; myocardial infarction; rehabilitation

\section{CARACTERIZACIÓN DE LA OBESIDAD EN PACIENTES QUE SUFRIERON INFARTO DEL MIOCARDIO}

Este trabajo intentó caracterizar la obesidad en pacientes infartados. Las estadísticas fueron recolectadas en 43 pacientes de un hospital universitario. Esas cifras muestran que el 69,7\% de los casos poseían un Índice de Masa Corporal

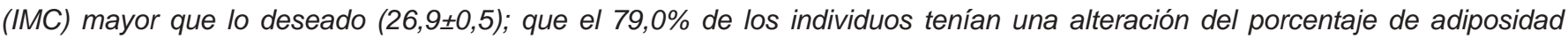
corporal $(20,0 \pm 1,0)$; que el $72,5 \%$ de los pacientes presentaban una relación cintura-cadera mayor que lo deseado $(0,97 \pm 0,03)$; y que, además, la circunferencia de la cintura (CC) estaba alterada en el $64 \%$ de los casos $(97,3 \pm 1,8)$. Como en el grupo estudiado la CC se correlaciona de modo significativo con el IMC y con el porcentaje de adiposidad corporal, se sugiere determinar el IMC y la CC para una evaluación de la obesidad en el momento de la consulta ambulatoria.

DESCRIPTORES: obesidad; infarto del miocardio; rehabilitación

\footnotetext{
${ }^{1}$ Enfermeira, Doutor em Enfermagem, Professor Colaborador da Faculdade de Ciências Médicas da Universidade Estadual de Campinas, e-mail: rcolombo@dgInet.com.br; ${ }^{2}$ Enfermeira, Doutor em Enfermagem, Professor Assistente da Escola de Enfermagem de Ribeirão Preto, da Universidade de São Paulo, Centro Colaborador da OMS para o desenvolvimento da pesquisa em enfermagem, e-mail: aguillar@eerp.usp.br; ${ }^{3}$ Enfermeira, Doutor em Enfermagem, Professor Colaborador da Faculdade de Ciências Médicas da Universidade Estadual de Campinas, e-mail: mcbjgallani@neuro.med.br; ${ }^{4}$ Professor de Educação Física, Doutor em Ciências Biológicas, Professor Assistente do Instituto de Biociências da Universidade Estadual de São Paulo, e-mail: cgobatto@rc.unesp.br
} 
INTRODUÇÃO

Em 1997, a obesidade foi reconhecida pela Organização de Mundial de Saúde como doença universal com importantes repercussões para a saúde pública ${ }^{(1)}$. De elevada prevalência em países desenvolvidos como Alemanha, Inglaterra e Estados Unidos ${ }^{(2)}$, a obesidade também apresenta índices crescentes em países em desenvolvimento. Um estudo ${ }^{(3)}$ recenterelatoua prevalência da obesidade na população adulta de 15 países (classificados em ricos, com níveis intermediários de riqueza e pobres de acordo com sua renda per capta, em 1991), com base nos resultados dos inquéritos probabilísticos de abrangência nacional, realizados no período de 1988-1996. Assim, considerando-se como nível crítico para obesidade o índice de massa corpórea (IMC) de $30 \mathrm{~kg} / \mathrm{m}^{2}$, verificou-se que Alemanha, Estados Unidos e Canadá apresentam prevalências elevadas de obesidade (entre 15 e $20 \%$ nos homens e entre 15 e $25 \%$ nas mulheres). No Brasil (classificado com nível intermediário de riqueza), a prevalência da obesidade mostrou-se elevada entre as mulheres $(13,3 \%$ entre as mulheres e $5,9 \%$ entre os homens). A comparação dos dados obtidos em três diferentes inquéritos de abrangência nacional, realizados em 1975, 1989 e 1996, evidencia o acelerado aumento da obesidade no Brasil, no período mais recente. Dessa forma, a prevalência da obesidade entre as mulheres que era de 4,4\% em 1975 aumentou para 10,1\%, em 1996. Entre os homens a prevalência da obesidade entre 0 período de 1975 e 1989 aumentou de 3,3\% para 5,9\% ${ }^{(2)}$.

A obesidade está associada ao incremento da mortalidade por inúmeras doenças crônicas, particularmente pela doença cardiovascular ${ }^{(4)}$. Sua importância como fator de risco, independente, para Doença Arterial Coronária (DAC) foi demonstrada com a divulgação dos resultados do estudo de Framingham ${ }^{(5)}$, quando ficou evidenciado que o ganho de peso durante a idade adulta aumenta o risco de DAC em ambos os sexos, independente da presença de outros fatores de risco.

Além de contribuir de forma isolada para a DAC, a obesidade é fortemente correlacionada aos fatores de riscos considerados primários para essa doença, destacando-se sua associação à hipertensão arterial sistêmica (HAS), diabetes mellitus e dislipidemia.

É importante ressaltar ainda a relação existente entre o padrão de distribuição da gordura corporal e o desenvolvimento e/ou progressão da DAC. O Professor
Jean Vague da Universidade de Marseille, França, na década de 40 , foi pioneiro ao demonstrar que o padrão de distribuição da gordura do tipo masculina, chamada obesidade andróide (predomínio da gordura no abdome e na parte superior do corpo), está associada a significativas complicações metabólicas, que se correlacionam com o desenvolvimento e/ou progressão da $\mathrm{DAC}^{(6)}$.

É comprovado, atualmente, que o excesso de gordura depositada na região abdominal é um forte preditor para doença cardiovascular e diabetes mellitus tipo 2. Isso pode ser parcialmente explicado pelo fato do excesso de acúmulo de gordura visceral relacionar-se, de forma independente à hipertensão $\operatorname{arterial}^{(7)}$, intolerância à glicose $^{(8)}$, resistência à insulina ${ }^{(9)}$ e à dislipidemia ${ }^{(9)}$.

A obesidade, portanto, se traduz em importante condição clínica que requer uma abordagem efetiva, especialmente no que se refere à prevenção primária e secundária da coronariopatia, onde a abordagem dos fatores de risco relaciona-se com o desenho de intervenções educacionais que possibilite ao sujeito a adoção de comportamentos positivos em saúde ${ }^{(10)}$. No entanto, a efetividade das intervenções voltadas para 0 controle desses fatores pressupõe, num primeiro momento, o conhecimento de como eles se distribuem na população a qual se destinam tais intervenções.

Assim, considerando-se a necessidade de modificação desse importante fator de risco, a descrição do padrão de obesidade do grupo de sujeitos, ao qual se destina um desenho de intervenções específicas, é de relevância. No contexto das doenças cardiovasculares, em especial da coronariopatia, essa descrição é muito importante, pois, nesse grupo, a prevalência dos fatores de risco, inclusive a obesidade, é maior que na população geral, e exige intervenção mais agressiva, bem como o controle periódico do resultado das intervenções implementadas. Quanto melhor caracterizada a obesidade, maior a especificidade da avaliação das intervenções.

Dessa maneira, este estudo teve como objetivo caracterizar a obesidade em relação às seguintes variáveis: IMC, percentagem de gordura corporal, relação cinturaquadril (RC/Q) e circunferência da cintura (CC), junto aos pacientes com infarto do miocárdio, atendidos num serviço de prevenção secundária. A caracterização detalhada dessas variáveis, além de proporcionar subsídios para o desenho de intervenções clínicas e educacionais e seu acompanhamento, fornece à literatura informações que permitirão comparar padrões de descrição da obesidade 
em outros grupos de sujeitos portadores de doenças cardiovasculares, identificando similaridades e peculiariedades entre eles.

\section{METODOLOGIA}

O trabalho foi desenvolvido no Ambulatório de Controle de Fatores de Risco para Coronariopatia (AMFACOR), que desde a sua criação em 1995, está vinculado ao Ambulatório de Cardiologia - subespecialidade de Cardiopatia Isquêmica, um dos ambulatórios coordenados pela Área de Cardiologia do Departamento de Clínica Médica da Faculdade de Ciências Médicas (FCM) da Universidade Estadual de Campinas (UNICAMP).

A amostra deste estudo foi constituída por 43 pacientes portadores de coronariopatia que apresentaram manifestação clínica de infarto do miocárdio, atendidos no AMFACOR, os quais atenderam os seguintes critérios de inclusão: capacidade de comunicar-se verbalmente e concordância em participar do estudo, oficializado através da assinatura do termo de consentimento livre e esclarecido. Destaca-se que este estudo foi aprovado pelo Comitê de Ética em Pesquisa da FCM/UNICAMP.

Os dados relativos à caracterização demográfica do grupo estudado foram obtidos através de um roteiro de entrevista, construído com perguntas fechadas. Esse roteiro foi submetido a pré-teste junto a dez sujeitos, procedendo-se a algumas alterações para sua melhor adequação. Para caracterizar a obesidade no grupo estudado, foram levantados dados referentes à composição corporal através do emprego da antropometria, de acordo com metodologia proposta em estudo pregresso ${ }^{(11)}$. Foram mensurados o peso, a altura, as circunferências (braço, cintura e quadril), as pregas cutâneas (tricipital, subescapular, supra-ilíaca) em homens e mulheres, a prega abdominal em homens e a prega da coxa em mulheres.

Através da mensuração do peso e altura dos sujeitos foi determinado o IMC, expresso pela relação peso $(\mathrm{kg}) /$ altura $^{2}(\mathrm{~m})$, cujos valores foram comparados com aqueles de referência adotados pelo comitê de juízes da Organização Mundial de Saúde ${ }^{(1)}$. De acordo com essa classificação é considerado saudável o valor de IMC entre 18,5 - 24,9; sobrepeso, 25,0 -29,9; obesidade grau I 30,0 - 34,9; obesidade grau II 35 - 39,9 e obesidade grau III 40,0 ou mais.
Para análise dos dados referentes à CC dos participantes deste estudo utilizou-se classificação proposta em estudo pregresso ${ }^{(12)}$, que categoriza os valores obtidos em níveis de ação 1 (homem: 94 cm; mulher: 80 cm) e 2 (homem: $102 \mathrm{~cm}$; mulher: $88 \mathrm{~cm}$ ), os quais estão relacionados com os riscos cardiovasculares e determinação de ações preventivas. Em relação à RC/Q, foi considerado como valor desejável para homens uma $\mathrm{RCQ}<0,95$ e para mulheres $<0,80^{(12)}$.

As pregas cutâneas foram mensuradas com a finalidade de obter-se a porcentagem de gordura corporal do grupo estudado. Visando minimizar variações de medidas decorrentes da pouca habilidade do examinador, as medidas de prega cutânea e das circunferências foram realizadas por uma nutricionista, habituada ao emprego desse procedimento em sua prática diária.

A interpretação das medidas de espessura das dobras cutâneas foi realizada através do emprego de equações específicas para predição dos valores de densidade corporal com base em espessuras de dobras cutâneas, desenvolvidas em estudo pregresso ${ }^{(13)}$.

Com a finalidade de se obter resultados imediatos da porcentagem de gordura relativa ao peso corporal, utilizou-se tabelas de conversão ${ }^{(11)}$ dos valores de densidade corporal, preditos através das equações de regressão, em percentagem de gordura relativa ao peso corporal para homens e mulheres.

Os dados referentes à percentagem de gordura do grupo estudado foram comparados àqueles de referência propostos em estudo prévio ${ }^{(14)}$, que preconiza como valor desejável para homens um percentual de gordura entre 5 e $15 \%$ e para mulheres entre 15 e $25 \%$.

As variáveis relativas à caracterização demográfica dos pacientes, levantadas através de questões fechadas foram codificadas e/ou categorizadas e, então, transportadas para tabelas de freqüência que permitiram a realização da análise estatística do tipo descritiva.

Para comparar duas variáveis categóricas foi utilizado o teste exato de Fisher. Para verificar a relação entre duas variáveis contínuas foi calculado o coeficiente de correlação de Spearman.

\section{RESULTADOS E DISCUSSÃO}

Em relação à caracterização do grupo estudado, 
verificou-se que a maioria era do sexo masculino $(76,7 \%$ 33/43), com idade entre 39 e 76 anos, com maior concentração na faixa etária de 40 a 49 anos (37,2\%), casados (74,4\% - 32/43), com escolaridade de 1 a 5 anos $(58,1 \%-25 / 43)$, inativos $(60,4 \%-26 / 43)$ e provenientes da cidade de Campinas e região (93\% - 40/43).

No que se refere à obesidade, foi constatado que $72 \%$ dos sujeitos (30/43) apresentavam IMC maior que 0 desejado, com concentração na faixa correspondente ao sobrepeso, como apresentado na Tabela 1.

Tabela 1 - Distribuição dos 43 sujeitos estudados, segundo o IMC e o sexo. Campinas/SP, 1999

\begin{tabular}{|c|c|c|c|c|c|c|c|c|}
\hline \multirow{3}{*}{$\mathrm{IMC} \mathrm{Kg/ \textrm {m } ^ { 2 }}$} & \multicolumn{6}{|c|}{ SEXO } & \multirow{2}{*}{\multicolumn{2}{|c|}{ TOTAL }} \\
\hline & \multicolumn{3}{|c|}{ MASCULINO } & \multicolumn{3}{|c|}{ FEMININO } & & \\
\hline & $\mathrm{n}$ & $\%^{*}$ & $\%$ ** & $\mathrm{n}$ & $\%$ & $\%$ & $\mathrm{n}$ & $\%$ \\
\hline Peso saudável $(18,5-24,9)$ & 10 & 30,3 & 23,2 & 2 & 20,0 & 4,6 & 12 & 28,0 \\
\hline Sobrepeso $(25,0-29,9)$ & 16 & 48,4 & 37,2 & 6 & 60,0 & 13,9 & 22 & 51,1 \\
\hline Obesidade Grau I $(30,0-34,9)$ & 7 & 21,2 & 16,3 & 1 & 10,0 & 2,3 & 8 & 18,6 \\
\hline Obesidade Grau II $(35,0-39,9)$ & - & - & - & 1 & 10,0 & 2,3 & 1 & 2,3 \\
\hline Obesidade Grau III $(>40,0)$ & - & - & - & - & - & - & - & - \\
\hline TOTAL & 33 & 100 & 76,7 & 10 & 100 & 23,1 & 43 & 100 \\
\hline
\end{tabular}

* percentagem referente ao número de sujeitos do sexo masculino

** percentagem referente ao total de sujeitos

A principal limitação do IMC como método de estimativa da gordura corporal é que não possibilita a distinção entre massa gorda (tecido adiposo e gordura) e massa magra (componente isento de gordura, calculada a partir de água, mineral e matéria orgânica, incluindo-se a quantidade não determinada de lipídios essenciais), principalmente em idosos, em que a perda de massa magra contribui para aumentar a variação de peso ${ }^{(15)}$.

Assim, outro indicador utilizado para avaliar a gordura corporal no grupo estudado foi a percentagem de gordura corporal, obtida a partir da soma das dobras cutâneas e interpretada a partir de equações de regressões.

O fato de as espessuras das dobras cutâneas serem realizadas em tecido mole, leva à possibilidade de cada examinador individualmente divergir quanto à exata localização e definição dos pontos de medidas. A precisão e exatidão dessas medidas relacionam-se com o emprego rigoroso da técnica de medida, qualidade do instrumento (compasso) utilizado, além do domínio pelo avaliador da técnica a ser empregada no procedimento ${ }^{(11)}$.

A interpretação das medidas de espessura de dobras cutâneas pode levar a erros de predição associados ao emprego de equações para estimativa de porcentagem de gordura que são definidos em torno de 5\%. Portanto, é necessário considerar a necessidade de validação junto à população que se pretende estudar, uma vez que equações desenvolvidas junto às populações norte-americana, européia, entre outras, apresentaram vieses ao serem utilizadas junto a segmentos da população brasileira ${ }^{(11)}$.

Por esse motivo, neste estudo, utilizou-se equações para predição dos valores de densidade corporal com base em espessuras de dobras cutâneas desenvolvidas em estudo pregresso ${ }^{(13)}$ junto a segmentos da população jovem brasileira (homens e mulheres entre 18 e 30 anos, residentes na cidade de Santa Maria, no Rio Grande do Sul). Segundo os autores, os erros de estimativa produzidos pelas equações variaram entre 1,5 e 3\% em indivíduos de diferentes quantidades de gordura corporal, o que mostra que os valores de densidade corporal podem ser estimados com precisão dentro dos limites admissíveis, independente dos índices de adiposidade apresentados pelos avaliados.

Os valores referentes à percentagem de gordura do grupo estudado que se encontravam acima do limite desejável foram distribuídos em categorias (leve, moderada ou elevada), segundo a classificação proposta, em 1993, por National Institute of Diabetes and Digestive and Kidney Diseases-NIDDK ${ }^{(16)}$ e estão apresentados na Tabela 2.

Assim, observa-se que 34 dos 43 pacientes possuíam alteração no percentual de gordura que oscilou entre os graus leve, moderado e elevado. Desses 34 sujeitos, 53\% (18/34) encontravam-se nas categorias moderada e/ou elevada, enquanto 47,0\% (16/34) foram categorizados em grau leve. Ao avaliar-se essa variável, em relação ao sexo, verifica-se que somente os homens apresentaram percentual de gordura categorizada como elevada. 
Tabela 2 - Distribuição dos 34 sujeitos com percentual de gordura acima do desejado, segundo as categorias de percentagem de gordura e o sexo. Campinas/SP, 1999

\begin{tabular}{|c|c|c|c|c|c|c|c|c|}
\hline \multirow{2}{*}{$\begin{array}{c}\text { Categorias de percentagem de } \\
\text { gordura }\end{array}$} & \multicolumn{6}{|c|}{ SEXO } & \multicolumn{2}{|c|}{ TOTAL } \\
\hline & $\mathrm{n}$ & $\%+$ & $\%++$ & $\mathrm{N}$ & $\%$ & $\%$ & $\mathrm{~N}$ & $\%$ \\
\hline Leve* & 13 & 48,1 & 38,2 & 3 & 42,8 & 8,8 & 16 & 47,0 \\
\hline Moderada** & 11 & 40,7 & 32,3 & 4 & 57,4 & 11,7 & 15 & 44,2 \\
\hline Elevada*** & 3 & 11,1 & 8,8 & - & - & - & 3 & 8,8 \\
\hline TOTAL & 27 & 100 & 79,3 & 7 & 100 & 20,5 & 34 & 100 \\
\hline
\end{tabular}

* homens: 15-20\%; mulheres: 25-30\%; ** homens: 20-25\%; mulheres: 30-35\%; *** homens: 25-30\%; mulheres: 35-40\%

+ percentagem referente ao número de sujeitos do sexo masculino

++ percentagem referente ao total de sujeitos

Além do IMC e do percentual de gordura utilizados na avaliação do excesso de peso, cresce o interesse no emprego de medidas de circunferências, em especial a $C C$ e a $R C / Q$ em função da estreita relação que guardam com a gordura visceral intra-abdominal, considerada fator de risco potencial para doenças crônicas, independente da obesidade total. Destaca-se, no entanto, que tais medidas são utilizadas como indicadores indiretos, já que não fornecem estimativa precisa da gordura visceral, a qual pode ser obtida apenas através de tomografia computadorizada ${ }^{(15)}$.

O levantamento da $\mathbf{R} \mathbf{C} / \mathbf{Q}$, no grupo estudado, mostrou que dos 40 sujeitos analisados $72,5 \%(29 / 40)$ apresentavam valor acima do desejado. Desses, $52,5 \%$ (21/40) eram homens e $20 \%$ (8/40) eram mulheres. No entanto, verificou-se que, dentre as mulheres, $88,8 \%$ (8/ 9) apresentavam $R C / Q$ maior que 0,80 , porém, essa diferença não foi significativa entre homens e mulheres $(p=0,399$, teste exato Fisher) (Tabela 3).

Tabela 3 - Distribuição dos 40 sujeitos estudados, segundo a RC/Quadril de acordo com o sexo. Campinas/SP, 1999

\begin{tabular}{|c|c|c|c|c|c|c|c|c|}
\hline \multirow{2}{*}{$R C / Q \cup A D R I L$} & \multicolumn{4}{|c|}{ MASCUINO * } & \multicolumn{2}{|c|}{ FEMININO ** } & \multicolumn{2}{|c|}{ TOTAL } \\
\hline & $\mathrm{n}$ & $\%+$ & $\%++$ & $\mathrm{n}$ & $\begin{array}{l}\text { M IN } \\
\%\end{array}$ & $\%$ & $\mathrm{~N}$ & $\%$ \\
\hline Desejado & 10 & 32,2 & 25,0 & 1 & 11,1 & 2,5 & 11 & 27,5 \\
\hline A lterado & 21 & 67,7 & 52,5 & 8 & 88,8 & 20,0 & 29 & 72,5 \\
\hline TOTAL & 31 & 100 & 77,5 & 9 & 100 & 22,5 & 40 & 100 \\
\hline
\end{tabular}

* valor alterado: maior ou igual a 0,95 ; ** valor alterado: maior ou igual a 0,80

+ percentagem referente ao número de sujeitos do sexo masculino

++ percentagem referente ao total de sujeitos

\section{A RC/Q é amplamente reconhecida como medida} clínica para identificar e classificar pacientes com acúmulo de gordura abdominal. Entretanto, recentes estudos têm sugerido que a CC, isoladamente, também se correlaciona com a obesidade abdominal ${ }^{(1)}$.

Analisando os resultados de CC no grupo estudado observou-se valor médio de $97,3 \pm 1,8 \mathrm{~cm}$, sendo que $64,3 \%(27 / 42)$ dos sujeitos apresentaram CC $\geq 94$ entre os homens e $\geq 80$ entre as mulheres.

Em 1995, um estudo ${ }^{(12)}$, que utilizou a medida de $C C$ e sua correlação com o IMC e a $R C / Q$, constatou que a $\mathrm{CC} \geq 94 \mathrm{~cm}$ em homens e $\geq 80 \mathrm{~cm}$ em mulheres, associou-se com elevado IMC $\left(\geq 25 \mathrm{~km}^{2}\right)$ ou com baixo IMC, mas com elevada RC/Q $(\geq 0,95$ para homens $\mathrm{e} \geq$ 0,80 para mulheres). A CC $\geq 102 \mathrm{~cm}$ em homens ou $\geq 88$ $\mathrm{cm}$ em mulheres identificou sujeitos com IMC $\geq 30 \mathrm{~kg} / \mathrm{m}^{2}$ ou com baixo IMC, mas com elevada RC/Q. Assim, foi sugerido pelos autores a categorização de valores de CC em "níveis de ação", preconizando que homens com CC $\geq 94$ $\mathrm{cm}$ e mulheres com $\mathrm{CC} \geq 80 \mathrm{~cm}$ devem ser aconselhados para evitar o ganho de peso (nível de ação 1) e homens com $\mathrm{CC} \geq 102 \mathrm{~cm}$ e mulheres com $\mathrm{CC} \geq 88 \mathrm{~cm}$ devem receber orientação quanto à necessidade de perder peso (nível de ação 2).

Posteriormente, estudo(17), envolvendo ampla amostragem, validou os níveis de ação, previamente propostos, e avaliou, junto aos indivíduos arrolados para o estudo, a relação entre fatores de risco cardiovasculares e a CC, categorizada em níveis de ação. Seus achados mostraram estreita relação entre fatores de risco cardiovasculares e CC. Dessa forma, esses autores demonstraram que indivíduos com CC entre nível 1 e 2 (94-101 cm em homens e $80-87 \mathrm{~cm}$ em mulheres) apresentam uma prevalência dos demais fatores de risco cardiovasculares 1,5 a 2 vezes maior quando comparada à população total estudada. Aqueles que apresentam CC acima do nível de ação 2 apresentam prevalência 2,5 a 3 vezes àquela da população total. Com base nesses 
achados, os autores recomendam que mulheres com CC entre $80-87 \mathrm{~cm}$ e homens com CC entre $94-101 \mathrm{~cm}$, sejam orientados quanto à necessidade da manutenção do peso corporal e quanto à importância da mudança no estilo de vida, como, por exemplo, a intensificação da atividade física. Aqueles que apresentam valores acima do limite estabelecido para o nível 2 (CC>102 cm para homens e $\mathrm{CC}>88 \mathrm{~cm}$ para mulheres) necessitam de acompanhamento por profissionais de saúde com vistas à perda de peso, além da identificação e controle de outros possíveis fatores de risco.

Recentemente, estudo ${ }^{(18)}$, desenvolvido junto a pacientes coronariopatas de um serviço de reabilitação cardíaca, confirmou que a CC, categorizada em níveis de ação, pode ser utilizada para identificar pacientes adultos com elevado IMC e/ou com baixo IMC, mas com elevada $\mathrm{RC} / \mathrm{Q}$. O estudo demonstrou também que elevados níveis de CC em homens e mulheres com DAC estão associados com aumento no risco metabólico, a despeito do uso de terapêutica cardioprotetora. Os dados mostraram aumento no risco de hipertrigliceridemia em homens com $\mathrm{CC} \geq 94$ $\mathrm{cm}$ e em mulheres com $\mathrm{CC} \geq 88 \mathrm{~cm}$. Homens com CC acima do nível de ação 2 exibiram baixos valores de HDL- col (lipoproteína de alta densidade), elevados valores de triglicérides/ HDL-col e hipertensão diastólica.

Com a finalidade de verificar a associação entre fatores relacionados ao estilo de vida e níveis de ação da CC e IMC, foi realizado estudo ${ }^{(19)}$, o qual encontrou que o excesso de peso e a distribuição abdominal da gordura estão associados com aumento da idade, desemprego, inatividade física, consumo de álcool e tabagismo. Segundo os autores, cada fator de risco influencia a CC diferentemente e, que a compreensão dessas diferenças parece ser fundamental para nortear as intervenções em serviços de promoção de saúde.

Utilizando a classificação em níveis de ação, constatou-se que, dos 42 sujeitos que tiveram a CC mensurada, $50 \%(21 / 42)$ encontravam-se classificados acima do nível de ação $2 ; 14,2 \%$ (6/42) apresentavam valor de CC entre o nível de ação 1 e 2 e 35,71\% (15/42) apresentavam circunferência da cintura $<94 \mathrm{~cm}$ para homens e $<80 \mathrm{~cm}$ para mulheres. Ao analisar-se a distribuição dessa variável por sexo, verificou-se leve tendência das mulheres serem classificadas acima do nível 2 em relação aos homens ( $p=0,103$, teste exato de Fisher) como mostra a Tabela 4.

Tabela 4 - Distribuição dos 42 sujeitos estudados, segundo classificação da circunferência da cintura em nível de ação e o sexo. Campinas-SP, 1999

\begin{tabular}{|c|c|c|c|c|c|c|c|c|}
\hline \multirow{2}{*}{ CIRCUNFERÊNCIA DA CINTURA } & \multicolumn{6}{|c|}{ SEXO } & \multicolumn{2}{|c|}{ TOTAL } \\
\hline & $\mathrm{N}$ & $\%+$ & $\%++$ & $\mathrm{n}$ & $\%$ & $\%$ & $\mathrm{~N}$ & $\%$ \\
\hline A baixo do nível de ação $1^{*}$ & 13 & 40,6 & 30,9 & 2 & 20,0 & 4,7 & 15 & 35,7 \\
\hline Entre nível de ação 1 e $2^{* *}$ & 6 & 18,7 & 14,3 & - & - & - & 6 & 14,3 \\
\hline A cima do nível de ação $2^{* * *}$ & 13 & 40,6 & 30,9 & 8 & 80,0 & 19,5 & 21 & 50,0 \\
\hline TOTAL & 32 & 100 & 76,1 & 10 & 100 & 23,8 & 42 & 100 \\
\hline
\end{tabular}

* homens: < 94cm; mulheres: < 80cm; ** homens: $94-101 \mathrm{~cm}$; mulheres: $80-87 \mathrm{~cm}$; ${ }^{* *}$ homens: maior ou igual a $102 \mathrm{~cm}$; mulheres: maior ou igual a $88 \mathrm{~cm}$

+ percentagem referente ao número de sujeitos do sexo masculino

++ percentagem referente ao total de sujeitos

Em relação às variáveis antropométricas, o IMC apresentou correlação positiva significativa com a CC e com o percentual de gordura corporal. A CC, por sua vez, apresentou significativa correlação com a percentagem de gordura corporal e a RC/Q (Tabela 5).

Tabela 5 - Coeficientes de correlação de Spearman para variáveis antropométricas - IMC, CC, percentagem de gordura e $R C / Q$ e $C C$, percentagem de gordura e $R C / Q$. Campinas/SP, 1999

\begin{tabular}{|c|c|c|c|}
\hline & $\mathrm{CC}$ & Percentagem de gordura & $\mathrm{RC} / \mathrm{Q}$ \\
\hline IMC & $\begin{array}{c}0,83^{* *} \\
(42)^{*}\end{array}$ & $\begin{array}{c}0,72^{* *} \\
(43)\end{array}$ & $N S^{* \star *}$ \\
\hline $\mathrm{CC}$ & -- & $\begin{array}{c}0,64^{* *} \\
(42)\end{array}$ & $\begin{array}{c}0,57^{\text {** }} \\
(40)\end{array}$ \\
\hline
\end{tabular}

*Corresponde ao número de sujeitos; ** Significativo $(p<0,05) ;{ }^{* * *}$ Não significativo
Destaca-se que as variáveis antropométricas IMC, CC e percentagem de gordura - correlacionaram-se positiva e significativamente com o número de fatores de risco associados.

Assim, constatou-se, no grupo estudado, correlação positiva entre índices referentes à quantidade e à distribuição de gordura com o número de fatores de risco associados, ou seja, quanto maior o número de fatores de risco associados, maiores os valores de IMC, $\mathrm{CC}$ e de porcentagem de gordura corporal. Tais achados apontam para a complexa relação existente entre a obesidade (ou o sobrepeso) e outras co-morbidades.

Dados epidemiológicos e metabólicos têm demonstrado que a obesidade contribui para um grande número de condições clínicas: resistência à insulina, 
intolerância à glicose, diabetes mellitus, hipertensão, dislipidemia, dentre outros, além de haver tendência, cada vez maior, de considerar a obesidade como fator de risco independente para cardiopatia. A influência da obesidade sobre as cardiopatias e outras co-morbidades está aumentada quando o excesso de gordura corpórea se dá predominantemente no abdômen e no tronco. É destacado, entretanto, que muito ainda tem que ser elucidado sobre os mecanismos subjacentes e sobre os aspectos quantitativos da associação entre obesidade e suas comorbidades $^{(20)}$.

A literatura aponta para a importância da obtenção de pelo menos dois métodos para análise da gordura corporal: um para quantidade (IMC ou percentagem de gordura corporal) e outro para avaliação de sua distribuição (RC/Q ou CC). Considerando-se que a mensuração da CC guardou correlação fortemente positiva com os dois índices de quantificação (IMC e porcentagem de gordura corporal), o que não foi observado para a $R C / Q$, nesse grupo estudado, parece interessante sugerir que sejam adotadas as mensurações de IMC e de CC para avaliação

\section{REFERENCIAS BIBLIOGRAFICAS}

1. Word Health Organization. Obesity: preventing and managing the global epidemic. Report of a WHO consultation on obesity. Geneva, June 3-5, 1997. Geneva:WHO; 1998.

2. Zanella MT. Obesidade. In: Mion D-Junior, Nobre F. Risco Cardiovascular Global. São Paulo (SP): Lemos Editorial; 1999. p.103-14

3. Monteiro CA. Epidemiologia da obesidade. In: Halpern A, Matos AFG, Suplicy HL, Mancini MC, Zanella MT, organizadores. Obesidade. São Paulo (SP): Lemos Editorial; 1998. p.15-30. 4. Montaye M, Bacquer DD, Backer DG, Amouyel P. Overweight and obesity: a major challenge for coronary heart disease secondary prevention in clinical practice in Europe. Eur Heart J 2000; 21(10):808-13.

5. Hubert HB, Feinleib M, McNamara PT, Castell WP. Obesity as an independent risk factor for cardiovascular disease: a 26-years follow-up of participants in the Framingham Heart Study. Circulation 1983; 67(5):968-77.

6. Depres JP, Moorjani S, Lupien PJ, Tremblay A, Nadeau A, Bouchard C. Regional distribution of body fat, plasma lipoproteins, and cardiovascular disease. Arteriosclerosis 1990; 10(4):497-511.

7. Peiris AN, Sothmann MS, Hoffmann RG, Hennes MI, Wilson CR, Gustapson AB, et al. Adiposity, fat distribution, and cardiovascular risk. Ann Intern Med 1989; 110:867-72.

8. Peiris AN, Struve MF, Mueller RA, Lee MB, Kissebah AH. Glucose metabolism in obesity: influence of body fat distribution. J Clin Epidemiol 1988; 67:760-7.

9. Depres JP Visceral obesity, insulin resistance and dyslipidemia: contribution of endurance exercise training to the treatment of the plurimetabolic syndrome. Exercise Sport Sci Rev 1997; 25:271-300. da gordura corporal, como práticas rotineiras no atendimento periódico.

\section{CONCLUSÃO E CONSIDERAÇÕES FINAIS}

Os resultados deste estudo demonstram que a obesidade destacou-se como significativo fator de risco no grupo estudado. Constatou-se que $69,7 \%$ dos sujeitos (30/43) possuíam IMC maior que o desejado $(26,9 \pm 0,5)$; $79 \%$ dos indivíduos (34/43) possuíam alteração no percentual de gordura corporal $(20,0 \pm 1,0) ; 72,5 \%$ dos sujeitos (29/40) apresentavam RC/Q acima do desejado $(0,97 \pm 0,03)$; a CC esteve alterada em $64 \%$ (27/42) dos sujeitos $(97,3 \pm 1,8)$. A correlação positiva da CC com os dois índices de quantificação de gordura corporal (IMC e percentagem de gordura corporal) e a praticidade para obtenção do IMC, leva a sugerir que a determinação do IMC e da CC seja adotada para avaliação e/ou controle da obesidade em pacientes infartados. Tais dados são fundamentais para subsidiar ações educativas voltadas para a prevenção secundária da coronariopatia.

10. Colombo RCR, Aguillar OM. Estilo de vida e fatores de risco de pacientes com primeiro episódio de infarto agudo do miocárdio. Rev Latino-am Enfermagem 1997; 5(2):69-82. 11. Guedes DP, Guedes JERP. Controle do peso corporal: composição corporal, atividade física e nutrição. Londrina (PR): Midiograf; 1998.

12. Lean MEJ, Han TS, Morrison CE. Waist circumference as a measure for indicating need for weight management. BMJ 1995; 311(15):158-61.

13. Guedes DP, Guedes JERP. Proposição de equações para predição da quantidade de gordura corporal em adultos jovens. Semina 1991:12:61-70.

14. McArdle WD, Katch FI, Katch VL Essential of exercise physiology. Malvern: Lea \& Febiger; 1994.

15. Willett WC, Dietz WH, Colditz GA. Guidelines for healthy weight. N Engl J Med 1999; 341(6):427-34.

16. National Institute of Diabetes and Digestive and Kidney Diseases. Understanding adult obesity. $\mathrm{NIH}-$ Publ. $\mathrm{n}^{\circ}$ 943680.Rockvilli, MD: National Institute of Health; 1993.

17. Han TS, Leer EMV, Seidell JC, Lean MEJ. Waist circumference action levels in the identification of cardiovascular risk factors: prevalence study in a random sample. BMJ 1995; 311(25):1401-5.

18. Carrol S, Cooke CB, Butterly RJ, Moxon JWD, Moxon JWA, Dudfield M. Waist circumference in the assessment of obesity and associated risk factors in coronary artery disease. Coronary Health Care 2000; 4(4):179-86.

19. Han TS, Bijnen FCH, Lean, MEJ, Seidell JC. Separate associations of waist and hip circumference with lifestyle factors. Int J Epidemiol 1998; 27: 422-3.

20. Grundy SM. Physical activity in the prevention and treatment of obesity and its comorbidities. Med Sci Sports Exerc 1999; 31(11):502-8. 